\title{
Transient heat transfer in rotating cylinders - Thermography measurement to analyse intense heat flux distributions
}

\author{
by J.C. Batsale*, J.P. Lasserre ${ }^{\star \star}$, M. Varenne - Pellegrini**, V. Desormiere ${ }^{\star \star}$, L. Autheserre ${ }^{\star \star}, A$. \\ Descuns $^{\star *}$, G. Lamothe ${ }^{\star *}$ \\ ** CEA, CESTA, F-33114 Le Barp, France. \\ *TREFLE-ENSAM, UMR 8508, Esplanade des Arts et Métiers, F-33405 Talence, France.
}

\section{Abstract}

The analysis of spatial distribution of intensive heat flux on curved surfaces is a crucial aspect of atmospheric re-entry characterization. This issue can be studied with ground testing facilities. This work is devoted to exploring several possibilities offered by experiments where a rotating cylinder is exposed to an intense heat flux. The first step of the experiment consists in analysing the temperature response measured by IR thermography on the outer face of the cylinder, from a pulse heating on such face. The estimation of the thermal diffusivity of the sample and the initial temperature distribution are successively presented.

\section{Introduction}

The heat transfer analysis of a rotating cylinder exposed to intense heat flux can be studied by IR thermography on the outer face of the cylinder (see figure 1). The main advantage is to avoid exposure of the IR camera to the intensive flux environment and to allow indirectly a front face analysis.

Such temperature response contains information related to the radial heat diffusion, to the rotation rate of the system (occurrence of the slots) and the angular diffusion (erosion of the slots). It is also possible to process such temperature response in order to estimate the initial temperature distribution directly related to the shape of the heat flux delivered to the cylinder (crucial for the calibration of the test facility).

The analytical model of this experimental situation will be first examined especially in the case of the impulse response. The main point is then related to the separability of heat transfer allowing to simply explore different possibilities of parameters estimation (velocity, thermal diffusivity, initial temperature related to the heat flux).

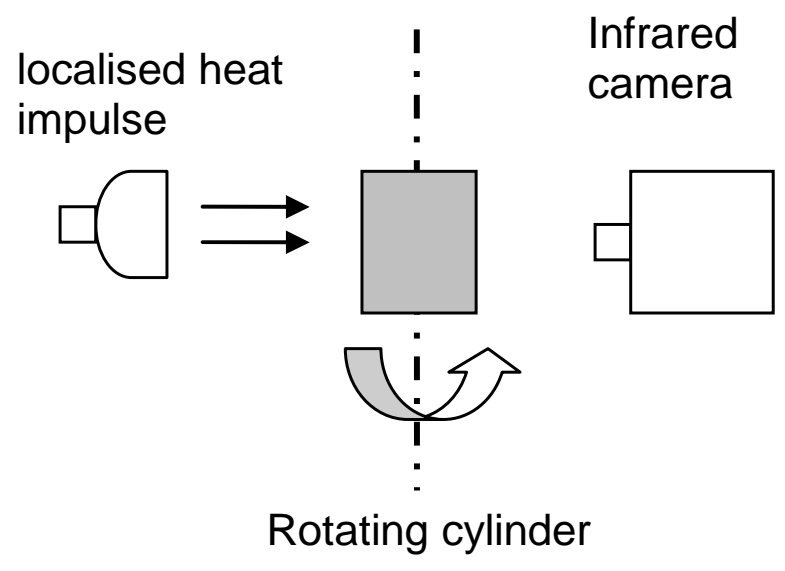

Fig 1: Experimental device 


\section{Analytical model}

A cylinder, rotating with a constant velocity receives a localised flash excitation at initial time (see the Fig 1). The temperature of the surface is measured in a non-moving laboratory coordinate system.

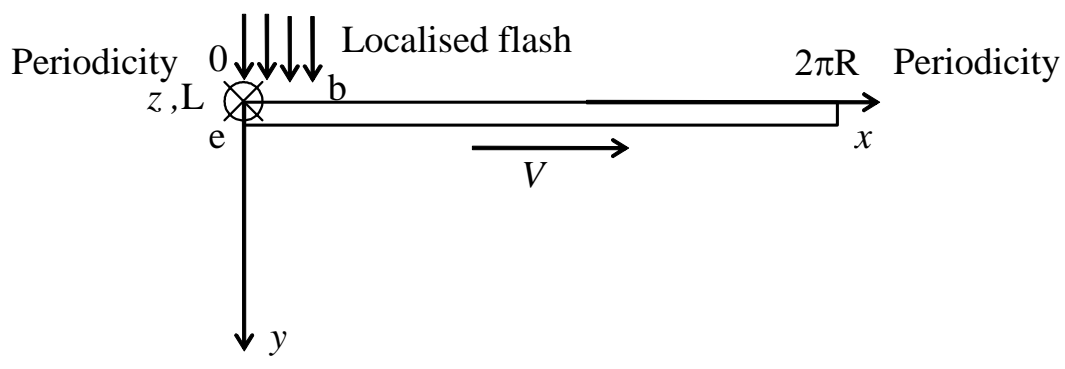

Fig 2: Simplified system from the figure 1 experiment.

The thickness of the hollow cylinder is assumed to be sufficiently small compared to the radius $R$ in order to consider the transfer in rectangular coordinates as shown in Fig. 2. When the transverse diffusion is assumed to be instantaneous, a general form of the temperature response is then a product of three terms such as (see Maillet et al [1]):

$$
\begin{aligned}
& T(x, y=0, t)=\frac{Q}{\rho c e}(1-\exp (-h t /(\rho c e))) \\
& \times\left(\frac{1}{2 \pi R} \sum_{-\infty}^{+\infty} \frac{1}{J \alpha_{n}}\left(1-\exp \left(-J \alpha_{n} b\right)\right) \exp \left(J \alpha_{n} x+J \alpha_{n} V t-a \alpha_{n}{ }^{2} t\right)\right) \\
& \quad \times\left(\frac{c}{L}+\frac{2}{L} \sum_{m=1}^{+\infty} \frac{\sin \left(\beta_{m} c\right)}{\beta_{m}} \cos \left(\beta_{m} z\right) \exp \left(-a \beta_{m}{ }^{2} t\right)\right)
\end{aligned}
$$

with $\alpha_{n}=\frac{2 n \pi}{2 \pi R}$

and $\beta_{m}=\frac{m \pi}{L}$

A simple computation example, corresponding to expression (1) with a space averaging versus $z$-direction is implemented (see results on Fig. 3). Some erosion due to diffusion following the x-direction can be observed for long times. Such response contains information related to the rotation rate of the system (occurrence of the slots) and the angular diffusion (erosion of the slots).

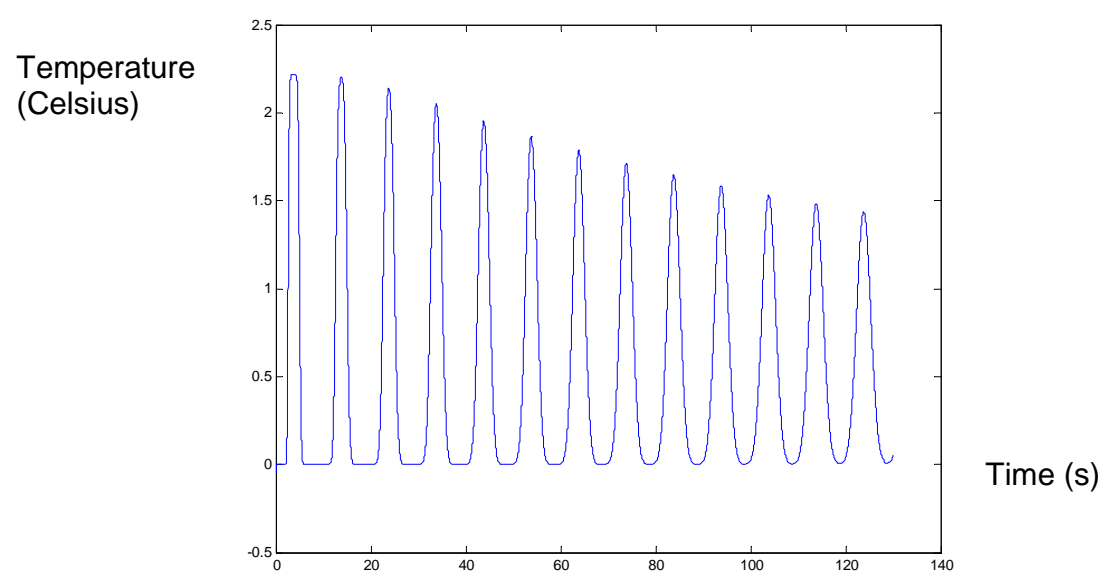

Fig 3: Example of theoretical temperature response (such a signal corresponds to the average of a column of pixels at a given position of the camera after a flash). 


\section{Experiment}

The experimental device is shown on fig 4. The heat source is obtained with a flash lamp which can deliver about $6400 \mathrm{~J}$ in about $4 \mathrm{~ms}$. In the experimental conditions (mask between the lamp and the rotating cylinder) such short pulse allows to study an equivalent continuous heat flux density which is about $20 \mathrm{MW} / \mathrm{m} 2$.

The thermographic device is a focal plane array camera with InSb detectors sensitive in the 3-5 $\mu \mathrm{m}$ infrared range. The scanning rate of the camera is 150 frames. On example of experimental temperature images is shown on fig 5 .

The rotating cylinder is made of stainless steel (of diffusivity $a=5.10^{-6} \mathrm{~m}^{2} \mathrm{~s}^{-1}$ ) of $10 \mathrm{~cm}$ in diameter. The rotation frequency of the cylinder is controlled by a step motor and is about $2 \mathrm{~Hz}$.

One example of complete experimental result which can be compared to the theoretical curve of figure 3 is shown on figure 6 .

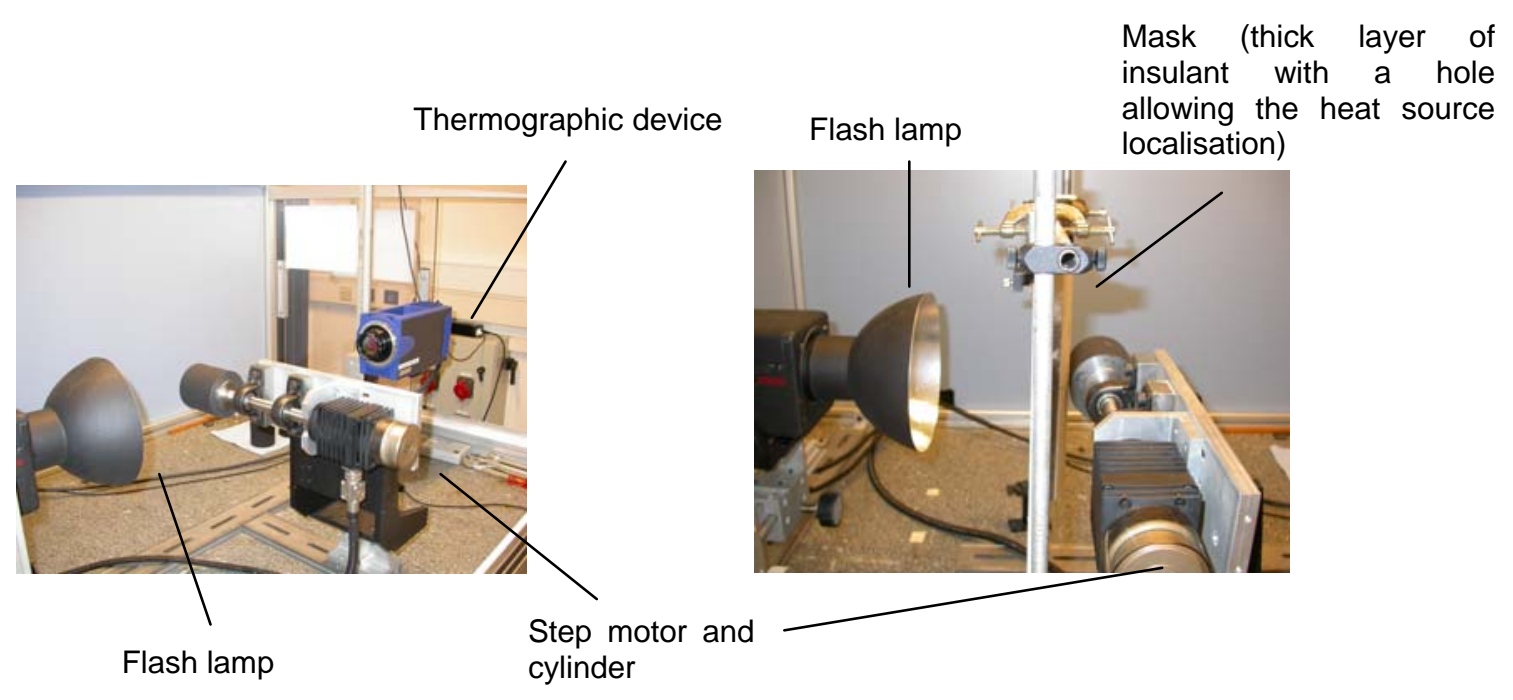

Fig4: Experimental device.
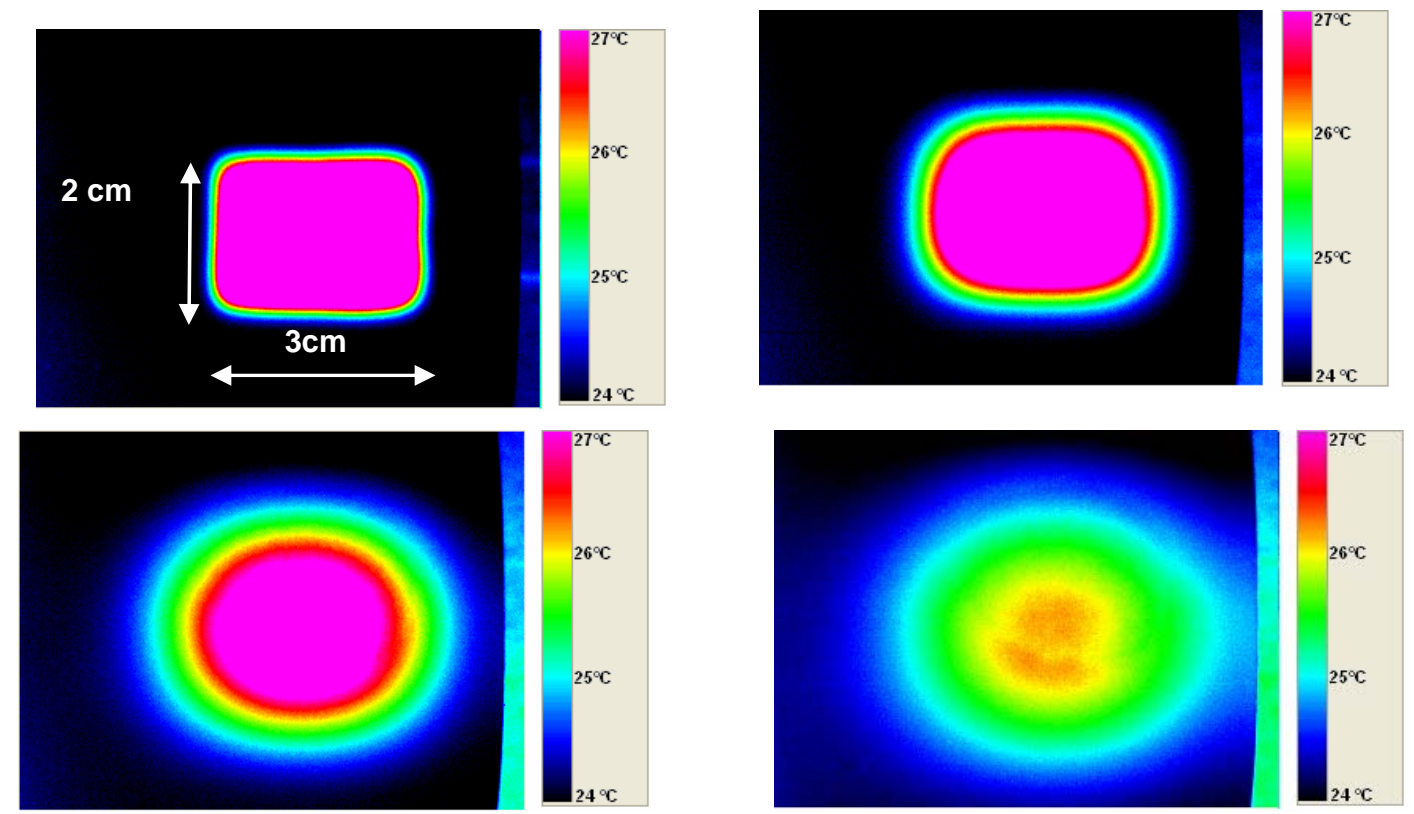

Fig 5 Examples of experimental temperature images at successive time $(0.5 ; 1.5,2.5,3.5$ revs). 


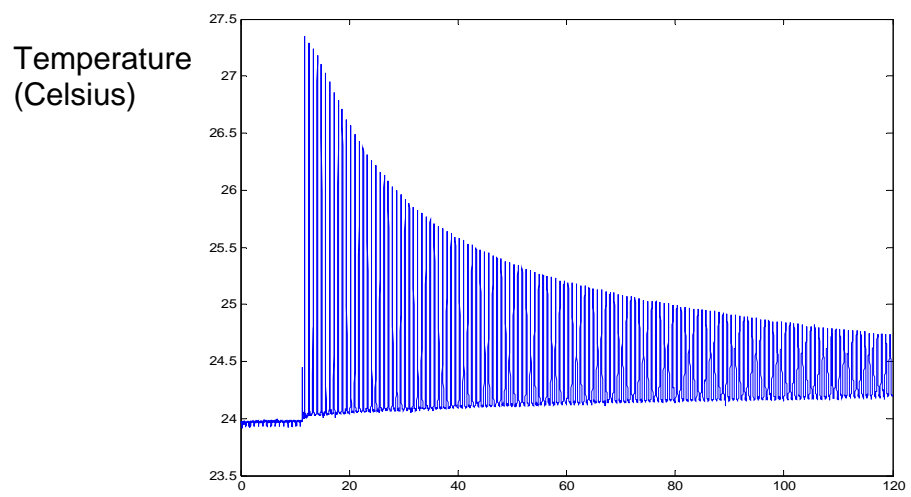

Time (s)

Fig 6: Experimental data obtained from the previous device and the column averaging on the temperature images (stainless steel sample).

\section{Data processing and parameters estimation}

\subsection{Velocity estimation}

The velocity parameter $V$ is obtained from data similar to those shown on figure 6 . Such velocity is assumed to be perfectly constant during the whole experiment. First, the Power Spectral Density (see figure 7) is computed in order to estimate a rough value of the rotation period. The maximum of the peaks localized inside the intervals previously defined are assumed to be related to an accurate location on the cylinder.

Then the velocity is estimated by considering the mean position of the maxima of the successive peaks shown on the signal versus the rotation time. The accuracy of the velocity estimation is observed (from the standard deviation) to be about $0.01 \mathrm{~Hz}$ for a rotation of about $2 \mathrm{~Hz}$. Such process is tested and validated on theoretical results.

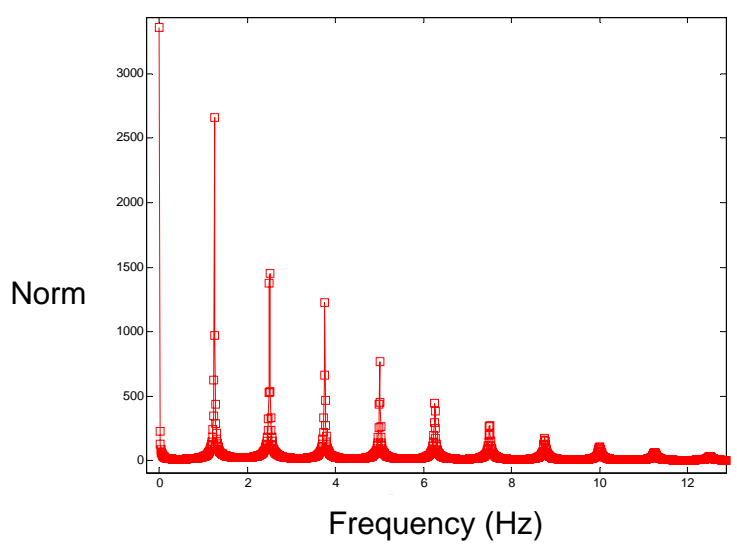

Fig 7. Power spectral density computed from the signal shown on Fig 6 in order to estimate the velocity parameter. 


\subsection{Diffusivity estimation versus $x$-direction}

The successive peaks are then superimposed thanks to the previous velocity estimation, because the successive time intervals are assumed to be related to the same repetitive cylinder location. Such other representation of the data is shown on figure 8.

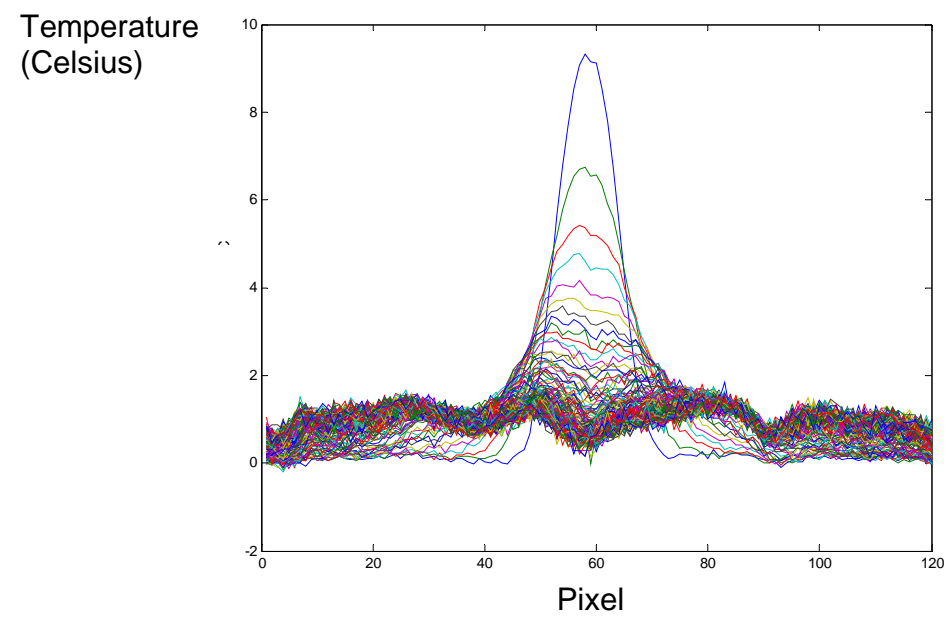

Fig 8. Superposition of the peaks from the rough data shown on figure 6 using the velocity estimation.

The thermal diffusion is eroding the signal at each time step and the Fourier transform versus time of such signal is such as:

$\theta\left(\alpha_{n}, t\right)=\sum_{i=1}^{N p i x} T(i, t) \exp \left(j \frac{n \pi i}{N p i x}\right)$

It allows then to write between two images at times $t_{1}$ and $t_{2}$ :

$\theta\left(\alpha_{n}, t_{1}\right)=\theta\left(\alpha_{n}, t_{2}\right) \exp \left(\left(-a \alpha_{n}^{2}-h /(\rho c e)\right) \cdot\left(t_{1}-t_{2}\right)\right)$

Such processing method is well known with non moving plate in order to estimate the thermal diffusivity of orthotropic composite samples (see [2] or [3])

The estimation of the diffusivity parameter is then possible by considering the slope of $\operatorname{Ln}\left(\theta\left(\alpha_{n}, t\right) / \theta\left(\alpha_{n}, 0\right)\right)$ versus time.

Unfortunately the real signal is not always corresponding to the theoretical one. A lot of precautions have to be considered in order to obtain such characteristics curves. In a first step, the slight emissivity variations of the painting (on the cylindrical sample) can superpose periodic noise to the temperature signal. Such variations are assumed to be smaller than the temperature evolution and subtracted to the real signal. The estimation of such fluctuations is made before the flash, when the sample is assumed to be isothermal.

A second important perturbation is caused by parasitic heating sources which do not allow to assume that the flash is the only source. Radiative effects due to the flash lamp or constant reflexions from the environment can modify the base line of the signal, which is increasing instead of remaining constant. A numerical process consisting in estimating at each time step the base line level of the signal must then be implemented.

One way to estimate the base line deviation is to select a few pixels far from the temperature bump and to locally estimate such level. The signal is then processed at each interval by considering a new base line. When the previously mentioned corrections are implemented, the estimation curves are then straight lines giving a good insurance about the diffusivity estimation (see Fig 9). In the case of the stainless steel sample, the estimated diffusivity by such a method is about $5 \%$ of the measure obtained with a standard flash method. 


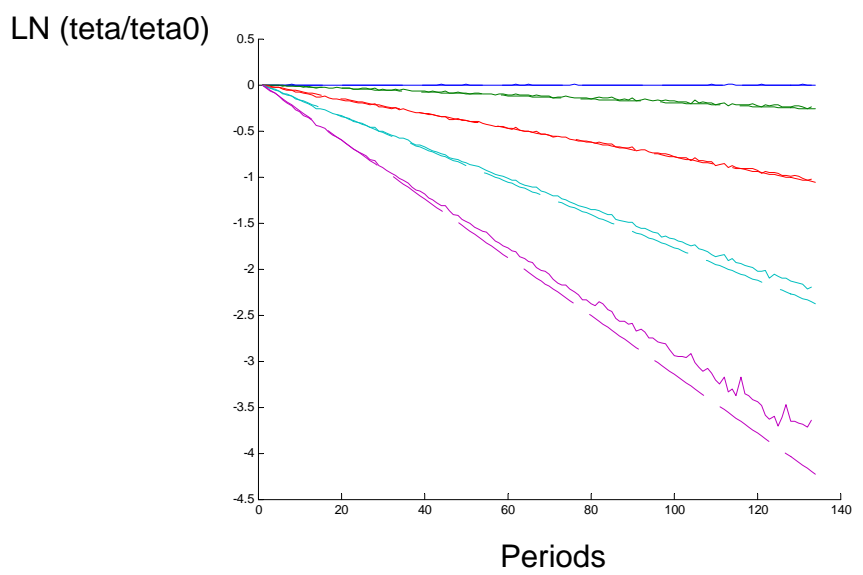

Fig 9: Theoretical and experimental curves for the diffusivity estimation.

\subsection{Diffusivity estimation versus $z$-direction}

A similar analysis can be implemented by considering the averaged signal $T_{r o t}(z, t)$ at each rotation of the cylinder by considering the following expression:

$T_{\text {rot }}(z, t)=\frac{1}{\tau} \int_{n \tau}^{(n+1) \tau} T\left(x=x_{0}, y=0, z, t\right) d t$

where $\tau=\frac{2 \pi R}{V}$ is defined as the duration of one rotation of the cylinder.

It is then possible to estimate the thermal diffusivity versus the $z$-direction by the same method as previously (expression (3)), with a cosine Fourier transform such as:

$\theta\left(\beta_{m}, t\right)=\sum_{i=1}^{\text {Mpix }} T(i, t) \cos \left(\frac{m \pi i}{M p i x}\right)$

The isotropy of the steel cylinder can here be verified. The estimation of the diffusivity is such as:

$a=(4.1 \pm 0.2) 10^{-6} \mathrm{~m}^{2} \mathrm{~s}^{-1}$

Such values are corresponding to the values measured with classical flash methods and referenced samples. Nevertheless, the main objective is not here to develop a new diffusivity measurement device, but to be able to estimate the initial temperature distribution related to the heat flux distribution.

\subsection{Estimation of the initial temperature distribution}

When the thermal diffusivity is known and the lateral heat losses assumed to be negligible, the previous expression (3) can be used in order to estimate the initial temperature field. It yields directly:

$\theta\left(\alpha_{n}, \beta_{m}, t_{2}\right)=\theta\left(\alpha_{n}, \beta_{m}, t_{0}\right) \exp \left(\left(a \alpha_{n}^{2}+a \beta_{m}^{2}\right) \cdot\left(t_{0}-t_{2}\right)\right)$

Such expression is useful, especially when the initial temperature distribution is separable such as:

$\theta\left(\alpha_{n}, \beta_{m}, t_{0}\right)=\theta\left(\alpha_{n}, 0, t_{0}\right) \theta\left(0, \beta_{m}, t_{0}\right)$ 
Each marginal temperature distribution can be recovered by considering separately two 1D non dependant temperature distributions: $\theta\left(\alpha_{n}, 0, t\right)$ and $\theta\left(0, \beta_{m}, t\right)$.

Unfortunately, the measurement noise is amplified at high frequency by the exponential term in expression (6). In order to avoid such problem, the great amount of data given by the infrared camera can be simultaneously processed with the following expression obtained from linear least square minimization:

$\hat{\theta}\left(\alpha_{n}, 0, t_{0}\right)=\frac{\sum_{k=1}^{N t} \exp \left(-a \alpha_{n}{ }^{2}\left(t_{k}-t_{0}\right)\right) \theta\left(\alpha_{n}, 0, t_{k}\right)}{\sum_{k=1}^{N t} \exp \left(-2 a \alpha_{n}{ }^{2}\left(t_{k}-t_{0}\right)\right)}$

Such processing must also be applied on the $\beta_{m}$ frequency direction. A regularization procedure can be implemented if the measurement noise is important.

In the present case, the estimation results have been applied and are shown on fig 10 . In the case of a quite square initial spot, the initial distribution can be assumed to be separable and gives convenient results.

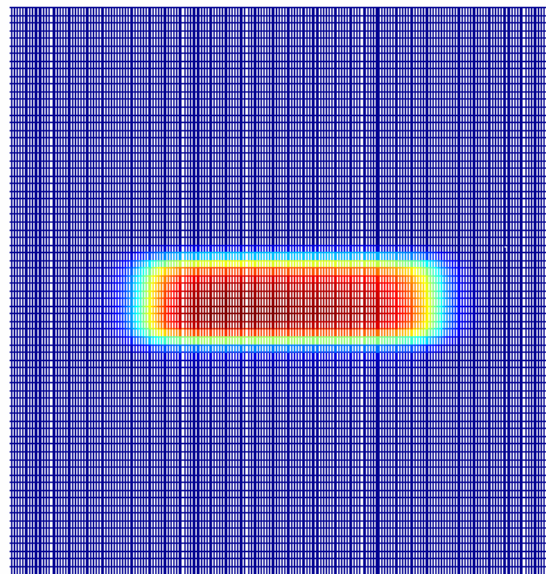

Temperature

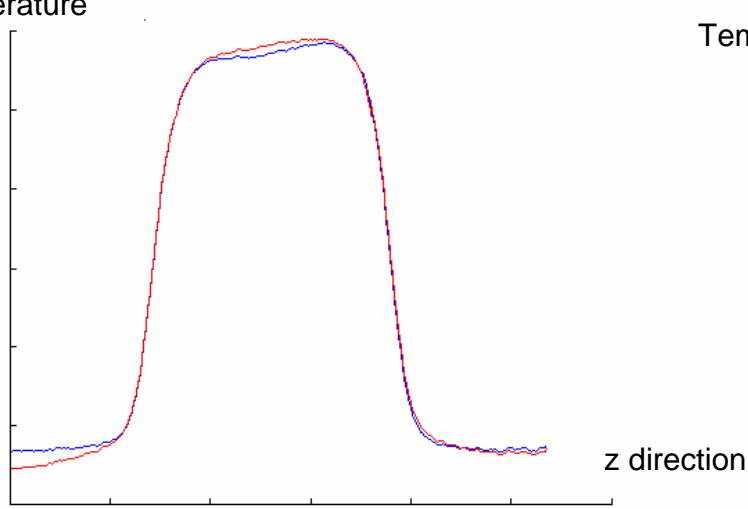

$x$ direction

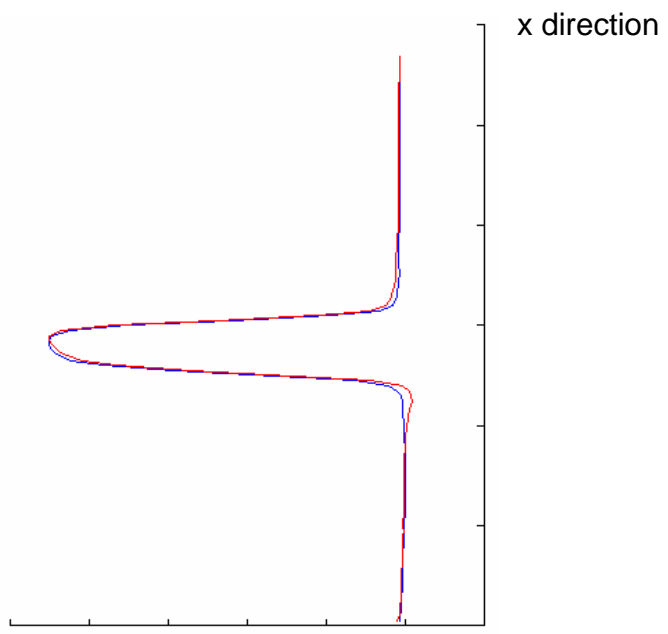

Temperature

Fig 10. Estimation of the $2 D$ initial temperature distribution

(red curve: estimation from the consecutive temperature observations, blue curve: measured initial distribution) 


\section{Conclusion}

The main advantages of the experimental method presented in this work is to be non intrusive in a case where severe conditions can be implemented and to be related to analytical models allowing simple considerations for the signal processing. The exploitation of the great amount of data given by the camera is then possible by using integral transforms such as Fourier transforms.

The intermediary step consisting in estimating the thermal diffusivity is important in order to test the bias of the model and to insure the initial temperature field estimation. The separability of the impulse temperature response allows to process a 2 or 3D heat transfer phenomenon with marginal 1D solutions obtained by considering spatial averaging.

Such preliminary steps are developed in the perspective to study continuous or variable heating conditions.

\section{References}

[1] D.MAILLET, S. ANDRE, J.C.BATSALE, A.DEGIOVANNI, C. MOYNE: Thermal quadrupoles- Solving the heat equation through integral transforms- J. Wiley Editor- Chichester- 2000, 270 pages, chap 5 (2000).

[2] I.PHILIPPI, J.C.BATSALE, D.MAILLET, A.DEGIOVANNI, 1995-Measurement of thermal diffusivity trough processing of infrared images -Rev. Sci. Instrum. 66(1) pp 182-192

[3] BISON P.G., GRINZATO E., MARINETTI S., Local thermal diffusivity measurement, QIRT Journal, 1, 2, pp 241-250. 